\title{
Homolytic Reduction of Onium Salts
}

\author{
Louis Fensterbank ${ }^{*}$, Jean-Philippe Goddard*, Max Malacria*, and Cyril Ollivier*
}

\begin{abstract}
Onium salts have proved to be efficient sources of carbon-centered radicals. They can undergo homolytic reduction by single electron transfer (SET) and participate in subsequent synthetic transformations. This review aims to provide an overview on the behavior of onium salts including diazonium, sulfonium, selenonium, telluronium, phosphonium and iodonium cations toward various reductive methods such as radiolysis, electrolysis, photolysis or the use of SET reagents. Mechanistic and synthetic aspects are presented. Applications in polymers and materials science are not covered.
\end{abstract}

Keywords: Diazoniums · lodoniums · Phosphoniums · Radicals · Reduction · Sulfoniums · Selenoniums · Telluroniums

\section{Introduction}

The chemistry of onium salts has attracted considerable interests and largely contributed to the development of organic chemistry over the past years. Such easily accessible species are important as precursors of ylides, leaving groups in substitution or elimination reactions, phase transfer catalysts and ionic liquid solvents.[1] Among numbers of applications, these structures could also serve as convenient sources of radicals under reductive conditions. In this review, diazonium, sulfonium, selenonium, telluronium, phosphonium and iodonium salts will be highlighted as efficient partners in homolytic processes. Different methods will be presented like electrochemical and photochemical reduction, radiolysis, but the focus will be on reactions featuring organic and organometallic single electron transfer reagents to promote homolytic transformations.

\section{Homolytic Reduction of Diazoniums}

Arenediazonium salts as precursors of aryl radicals have been extensively studied for more than a century. Based on the reduction of the diazonium group, different reactions have been reported in order to create carbon-heteroatom or carbon-carbon bonds (Scheme 1). In 1884, Sandmeyer proposed the transformation of aromatic and heteroaromatic amines into the corresponding aryl halides. ${ }^{[2,3]}$ The intermediate diazonium derivatives were obtained by treatment of the amine with sodium nitrite, and decomposed in the presence of copper(I) salts. Then the resulting aryl radical was trapped by the copper salt to afford the halogenated product. This reaction is still one of the most efficient to do this functional group transformation. A few years later, Pschorr showed the potential of arenediazonium decomposition for the formation of carbon-carbon bonds. ${ }^{[4]}$ In the presence of copper powder, judiciously defined substrates allowed the formation of phenanthrene derivatives by an

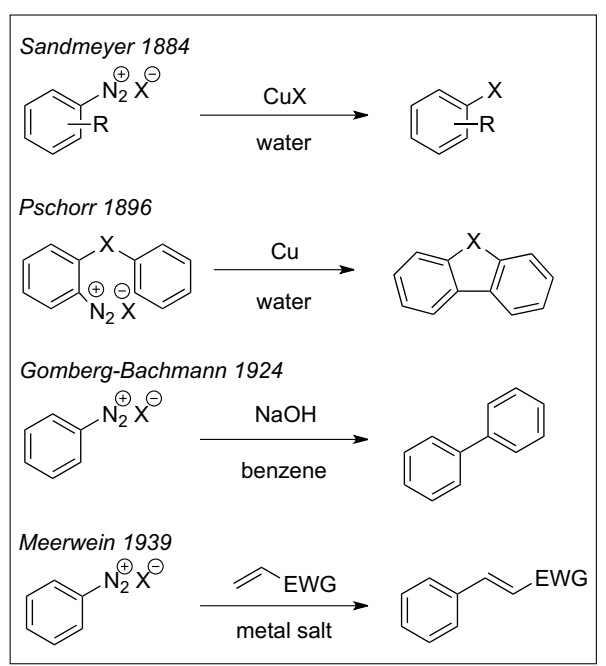

Scheme 1. intramolecular arylation. This reaction has been extended by Gomberg and Bachmann to an intermolecular arylation in order to prepare biphenyl compounds.[5] In 1939, Meerwein developed a related process where the aryl radical, generated by metalmediated decomposition of diazonium salt, reacted with an olefin as an acceptor. ${ }^{[6,7]} \mathrm{A}$ subsequent oxidation of the resulting radical afforded the corresponding Heck-type product. The kinetic parameters of these reactions have been studied by Kochi. ${ }^{[8,9]}$ Since these seminal works, a number of research groups have contributed to the development of new processes involving arenediazonium salts under reductive conditions. ${ }^{[10]}$ In this review, we will focus on processes that lead to the formation of carbon-carbon bonds and their applications in organic synthesis.

In a one-pot procedure, Doyle reported a variation of the Meerwein arylation reaction (Scheme 2). ${ }^{[11]}$ Aniline derivatives reacted with tert-butyl nitrite and copper(II) chloride in the presence of an electronpoor olefin. The arenediazonium salt was generated in situ and decomposed by the copper catalyst. The so-formed aryl radical added onto the acceptor to afford the corresponding alkyl radical that was oxidized by copper(II). This transformation led to the formation of the chlorinated compound in good yield. This convenient procedure avoids the formation of the Sandmeyer by-product that is usually obtained in the Meerwein reaction. Indeed, copper(II)

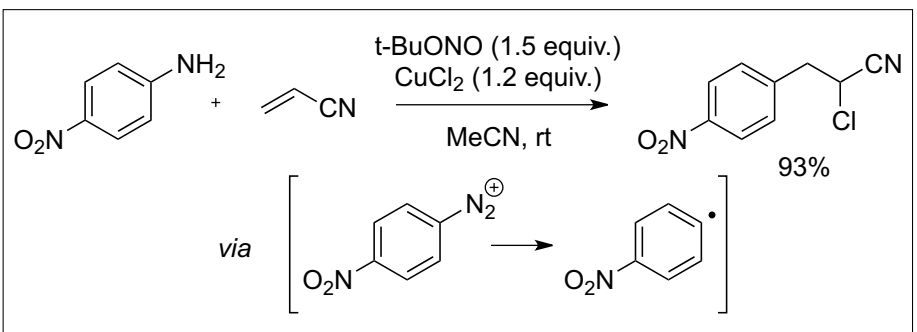




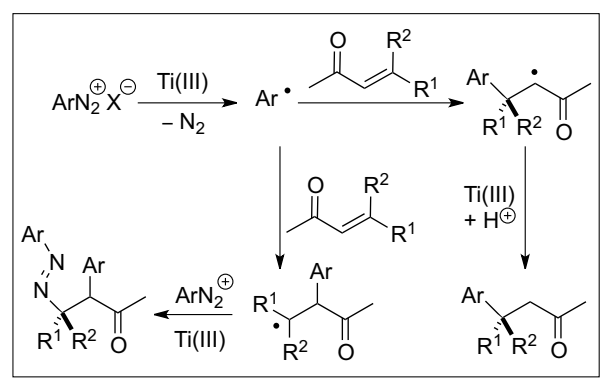

Scheme 3

if

Scheme 4

bromide was used to generate the bromide analog without traces of aryl bromide.

The reactivity and the regioselectivity of the aryl radical addition to $\alpha, \beta$ unsaturated carbonyl acceptors have been investigated by Citterio with aryldiazonium salts as radical precursors (Scheme $3)^{[12]}$ Titanium(III) chloride has been used for the reduction in acetic acid and water. The single electron transfer (SET) from Ti(III) to diazonium is known to be as fast as the decomposition of the resulting diazenyl radical.[13-15] Depending on the substitution profile of the olefin, the aryl radical either added at the $\alpha$ position of the carbonyl group or at the $\beta$ position. In the first case, the resulting radical was not quickly reduced in carbanion and reacted with a second diazonium molecule to form the diazene adduct. When the radical addition occurred at the $\beta$ position, the resulting radical was reduced and the arylation product was obtained.
Arenediazonium salts have also been used by Motherwell in the development of a tin-free intramolecular radical ipso substitution under reductive conditions (Scheme 4). ${ }^{[16]}$ Series of aryl sulfonamides and aryl sulfonates possessing a diazonium moiety at the ortho position have been engaged in SET transformations with titanium(III) chloride as optimal reductant. The aryl radical underwent an ispo substitution at the sulfur-substituted carbon center that was oriented by steric interactions. By the loss of sulfur dioxide and reduction, fully hindered biaryl compounds were accessed in good yields.

More recently, Heinrich revisited the chemistry of the arenediazonium salts as aryl radical precursors (Scheme 5). With $\mathrm{TiCl}_{3}$ or $\mathrm{FeSO}_{4}$ as SET reagents, diversely substituted diazonium derivatives allowed the formation of diazene compounds following the same mechanism that was evidenced by Citterio. ${ }^{[17]}$ The formal carboamination of the olefin was obtained after the hydrogenolysis of the diazene. In the presence of TEMPO ${ }^{[18]}$ or nitric oxide, ${ }^{[19]}$ the resulting radical adduct has been trapped to lead to the corresponding alkoxy tetramethylpiperidinyl and the oxime respectively. Allylation and vinylation of aryl radicals have been achieved with allyl and vinyl chloride as acceptors. ${ }^{[20]}$ By analogy with the Gomberg-Bachmann reaction, the same group reported the synthesis of amino- and hydroxybiphenyl derivatives promoted by titanium(III) chloride.[21]

More than a hundred years ago, Graebe and Ullman showed the formation of 9-fluorenone from the decomposition of 2-diazobenzophenone. ${ }^{[22]}$ Lewin and Cohen rationalized the different parameters that control this reaction. ${ }^{[23]}$ It has been demonstrated that thermal conditions in acidic medium generated the phenyl cation by heterolytic bond cleavage while the cor-
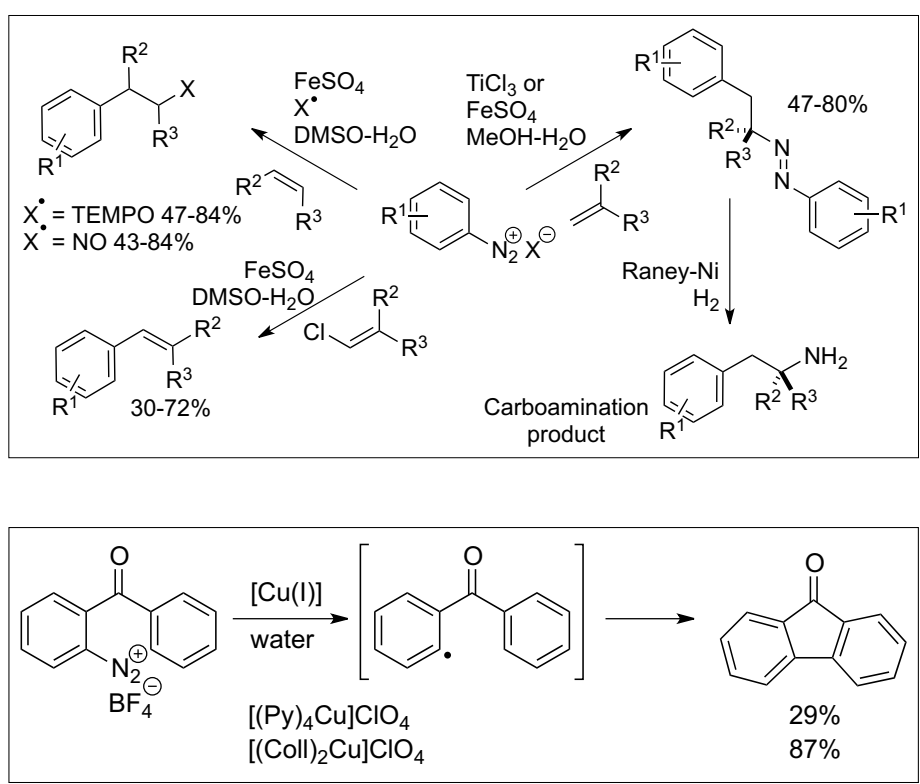

responding phenyl radical was obtained by the action of a SET catalyst. Amine copper(I) perchlorates proved to be effective catalysts for this homolytic process (Scheme 6).[24,25] 2-Diazobenzophenone tetrafluoroborate was used as model substrate. Depending on the aromatic amine ligand, it was possible to promote the aryl radical cyclization to afford 9-fluorenone among a number of by-products. For example, $\left[(\mathrm{Py}){ }_{4} \mathrm{Cu}\right] \mathrm{ClO}_{4}$ was poorly efficient (29\% of 9-fluorenone) and the major product originated from the dimerization of the intermediate aryl radical. By contrast, $\left[(\mathrm{Coll}){ }_{2} \mathrm{Cu}\right] \mathrm{ClO}_{4}$ allowed the cyclization in $87 \%$ yield. This illustrated the higher reducing ability of bis- and tris(amine) copper salts versus tetrakis(amine) copper complexes.

The Pschorr reaction has been nicely reported by Deronzier for the synthesis of phenanthrene derivatives involving a photocatalytic process (Scheme 7).[26] Under visible light irradiation, $\left[\mathrm{Ru}(\text { bipy })_{3}\right]^{2+}$ catalyst was photoexcited and became suitable for single electron transfer. Then, 2-diazo$\alpha$-arylcinnamic acid acted as an oxidative quench for the triplet ruthenium complex that was oxidized to $\mathrm{Ru}(\mathrm{III})$. At the same time, the diazonium was reduced to the corresponding aryl radical which cyclized onto the phenyl ring to form a cyclohexadienyl radical. The rearomatization occurred by an oxidation pathway catalyzed by the $\mathrm{Ru}(\mathrm{III})$ catalyst and allowed the regeneration of $\left[\mathrm{Ru}(\mathrm{bpy})_{3}\right]^{2+}$.

Thus, aryldiazonium salts can be considered as effective oxidative quenches for triplet ruthenium polypyridine complexes. In an original procedure, the same group reported the oxidation of primary and secondary alcohols into aldehydes and ketones respectively. ${ }^{[27,28]}$ The diazonium salt allowed the formation of $\mathrm{Ru}(\mathrm{III})$ that catalyzed the SET oxidation in the presence of a base. The catalytic process was managed by the $\mathrm{Ru}(\mathrm{III}) / \mathrm{Ru}(\mathrm{II})$ redox system. Sanford took advantage of these properties in order to merge palladium-catalyzed $\mathrm{C}-\mathrm{H}$ activation and redox photocatalysis (Scheme 8). ${ }^{229]}$ The diazonium salt was reduced by triplet $\mathrm{Ru}$ (II) catalyst to yield

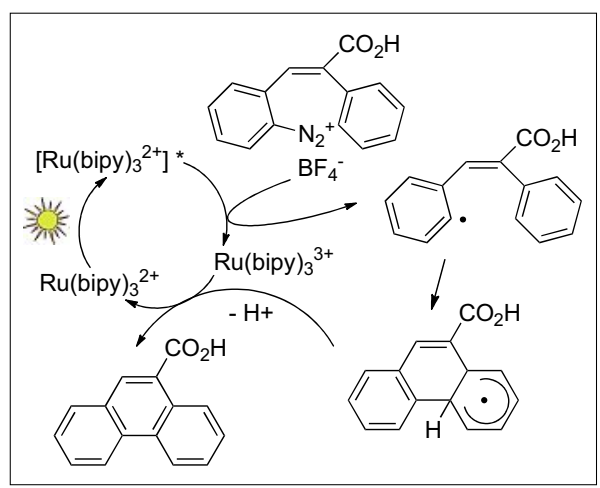

Scheme 7 
the corresponding aryl radical that added onto a Pd(II) complex to afford a Pd(III) intermediate. Oxidation by Ru(III) generated a $\operatorname{Pd}(\mathrm{IV})$ reactive entity which underwent reductive elimination to finish the catalytic cycle. Biaryl compounds were obtained in good yields.

Catalytic reduction of aryldiazonium salts was also reported in a metal-free process with Eosin $\mathrm{Y}$ as a catalyst (Scheme 9). [30] The SET process relied on this organic dye which was oxidized to the corresponding radical cation. The aryl radical underwent a Meerwein arylation reaction to afford biaryl compounds after rearomatization by the radical cation of EosinY.

Other neutral organic electron donors have been developed for the SET reduction of arenediazonium salts as precursors of aryl radicals. Murphy reported radical-polar crossover reactions of such substrates in splicing radical and polar reactions in the same pot with tetrathiafulvalene (TTF) or dithiadiazafulvalene (DTDAF) as reductants (Scheme 10). ${ }^{[31-34]}$ These processes have been applied to the total synthesis of Aspidospermidine ${ }^{35]}$ and number of other heterocycles such as indoles, indolines and dihydrobenzofurans. More recently, the same group reported the use of commercially available tetrakis(dimethylamino) ethylene (TDAE) as an alternative reagent for organic SET. ${ }^{[36]}$ Indolines have been obtained by a radical sequence involving a SET reduction of a diazonium salt to generate the corresponding aryl radical that cyclized according to a 5-exo-trig mode. A subsequent $\beta$-fragmentation allowed the formation of indolines and the liberation of a thiyl radical that dimerized. This very efficient transformation involved only one equivalent of the SET reagent at room temperature in only $5 \mathrm{~min}$ to obtain high yields of heterocyclic products.

The reduction of arenediazonium salts has been reported with other non-metallic reagents. In 1950, Cooper gave evidence for a free radical chain mechanism in the presence of hypophosphorous acid.[37] Arylhydrazines also proved to be efficient SET reagents for the initial reduction of the diazonium moiety but gave mixtures of products originating from aryl and diazenyl radicals. ${ }^{[38]}$ The iododediazoniation has been investigated to transform arendiazonium salt into the corresponding aryl iodide compound. While chloro- and bromodediazoniation needed appropriate cupric halide (Sandmeyer reaction), only alkali iodides allowed the aryl iodide formation. ${ }^{[39]}$ Mechanistic and kinetic studies have been performed by Beckwith and Abeywickrema in order to evidence a SET initiation from iodide to diazonium ion.

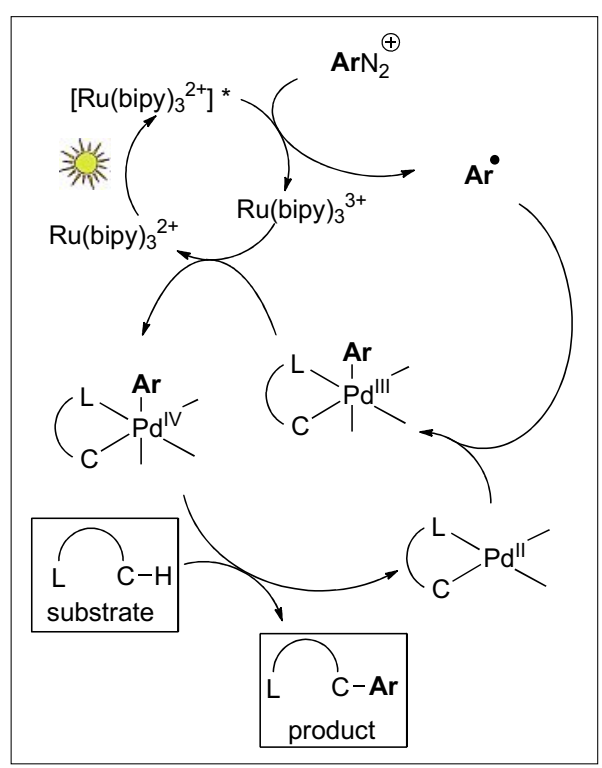

Scheme 8.

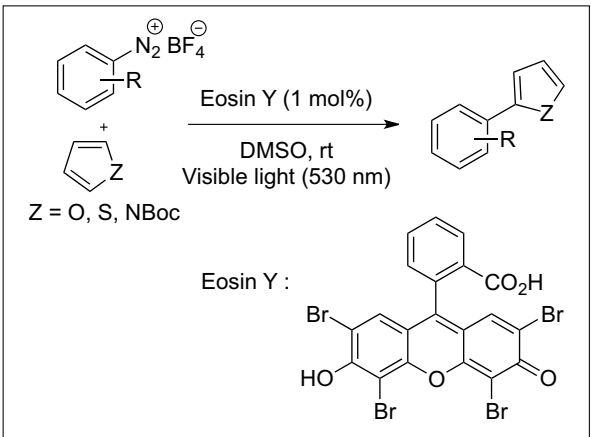

Scheme 9.

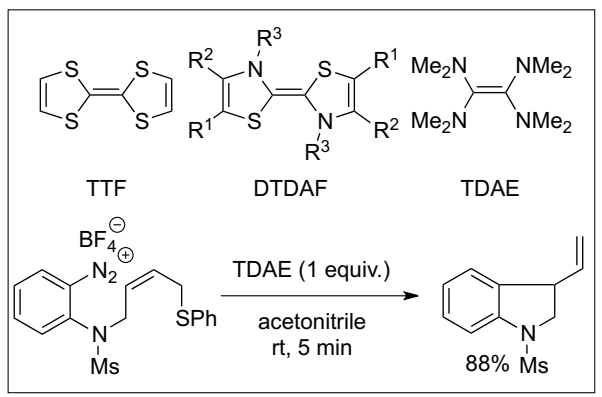

Scheme 10.

\section{Homolytic Reduction of Sulfoniums, Selenoniums, and Telluroniums}

Homolytic reduction of sulfoniums to generate $\mathrm{C}$-centered radicals are wellknown biochemical processes, as illustrated by the reduction of the sulfonium of S-adenosylmethionine (SAM) by ironsulfur clusters containing enzymes (SAM enzymes). ${ }^{[40,41]}$ It has also gained high importance in materials science notably for the functionalization of surfaces. ${ }^{[42]}$

Herein, we will limit our overview to molecular processes with relevance to organic synthesis. Interestingly, it appears that the above mentioned natural processes have not really constituted a source of in- spiration for the development of synthetic processes since the homolytic reduction of sulfoniums has been reported through different methods including radiolysis, electrolysis, photolysis or the use of single electron transfer (SET) reagents with no mention of the natural radical pathway and in some cases even before the discovery of the latter. We will cover this continuously active field by type of reactions in the order given above.

Thus, the use of solvated electrons produced in aqueous solution by radiolysis was initially shown by Bonifacic to be a very rapid and clean method to generate alkyl radicals from a variety of sulfoniums, including $S$-methylmethionine and dimethyl-b-propiothetin. ${ }^{[43]}$ This was further confirmed by Engman who studied the reduction of triorganylsulfonium, -selenonium and -telluronium salts and evidenced that the radical expulsion was dictated by the stability of the generated radical species (benzyl > secondary alkyl $>$ primary alkyl $>$ methyl $>$ phenyl). ${ }^{[4]}$

Electrolysis of sulfonium salts has been the object of several studies oriented toward synthetic applications ${ }^{[45]}$ or mechanistic considerations. ${ }^{[46]}$ In this context, the Savéant group has been particularly active in notably questioning the concerted or stepwise character of the electron transfer with the bond cleavage. They established that dimethylphenylsulfonium is electrochemically reduced in a stepwise process involving a radical anion intermediate. Logically, it was deduced from these studies that the concerted character is prevailing when the LUMO of the sulfonium is high and the bond to be broken is weak. ${ }^{[47]}$ In 2009, Kampmeier and Lund further refined this by reinvestigating some previous puzzling findings: monoaryldialkylsulfonium salts never give aryl radicals whereas diarylmonoalkyl result in aryl cleavage. ${ }^{[48]}$ They found that the regioselectivity of cleavage can be controlled by the potential of the reducing agent and deduced that the structure of the sulfuranyl radical intermediate and the bond dissociation energies of the alkyl and aryl substituents are the key parameters.

Photolysis of sulfoniums has been studied by several groups. ${ }^{[49]}$ Hacker showed that upon UV irradiation of triarylsulfoniums, rearrangement products such as phenylthiobiphenyls and diphenylsulfide are produced. These reactions involve predominantly heterolytic cleavage to generate a phenyl cation and diphenylsulide and as a minor homolytic pathway, a phenyl radical and a diphenylsulfide radical cation. [50] Interestingly, in the case of the charge transfer complex triphenylsulfonium iodide, a triphenylsulfuranyl radical intermediate is presumably formed upon photolysis that fractionates. ${ }^{[51]}$ This paved 
a way for the photoinduced reduction of sulfonium salts. Kampmeier examined the photosensitized reduction of 4-cyanobenzylmethylphenylsulfonium tetrafluoroborate by substituted anthracenes or perylene, ${ }^{[52]}$ while Saeva reported an intramolecular PET process. ${ }^{[53]}$ Interestingly, Kellogg in a very seminal work reported a Hantzsch ester derivative mediated reduction of phenylacylsulfonium salts in the presence of visible light (Scheme 11). This reaction could be accelerated thanks to photosensitive agents such as organic dyes and $\left[\mathrm{Ru}(\mathrm{bpy})_{3}\right]^{2+} \cdot{ }^{[54]}$

It is worthy of note that triarylsulfonium salts are reduced upon treatment with simple alkoxides such as sodium isopropoxide and potassium hydroxide. The intermediacy of radical species was established. ${ }^{[55]}$

In 1982, Beak investigated the reduction of various arylalkylsulfonium salts upon treatment with potassium in graphite. ${ }^{[56]}$ In a monophenyldialkylsulfonium series, the same leaving-group propensity as before (benzyl > secondary alkyl $>$ primary alkyl $>$ methyl $>$ phenyl) was found. A mechanism was proposed involving the transfer of one electron to the phenyl LUMO generating a $\pi$-radical anion. Cleavage then would take place through the overlap of the SOMO of the aromatic ring with the $\sigma^{*}$ orbital of the bond which falls apart homolytically. Interestingly, dimethyl-tert-butylsulfonium as well as methyltetramethylene sulfonium were not reduced in these conditions, presumably because there is no low-lying LUMO to accept an electron. In the case of diphenyl and benzo-fused salts, it was found that the leaving-group propensities differ from the one observed with the monophenyl salts.

The group of Hori and Kataoka has also studied the homolytic reduction of cyclic sulfonium and selenonium salts with magnesium metal or Grignard reagents and also with $\mathrm{SmI}_{2}$ which proved to a stronger but less regioselective reagent, as illustrated by the reductions of 2-methylisoselenochromanium salt shown below (Scheme 12). ${ }^{[57]}$

An interesting application was the reduction of the following tricyclic benzothiazinium salt which generates three types of products according to the reaction conditions used (Scheme 13). Unfortunately, no rationalization was given for these contrasting findings. ${ }^{58]}$

In the acyclic series, this process was applied to a cyclization process (Scheme 14). But clearly, poor regiocontrol in the homolytic cleavage plagues the versatility of the reaction. ${ }^{[57 \mathrm{~d}]}$

Although non preparative, an interesting redox radical chain reaction to reduce biaryliodoniums and triarylsulfoniums has been devised by Kampmeier (Scheme 15).

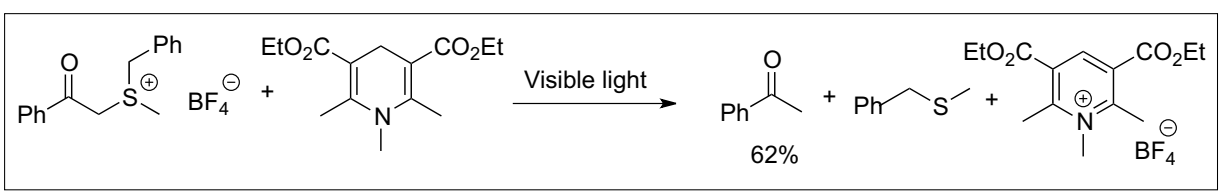

Scheme 11.

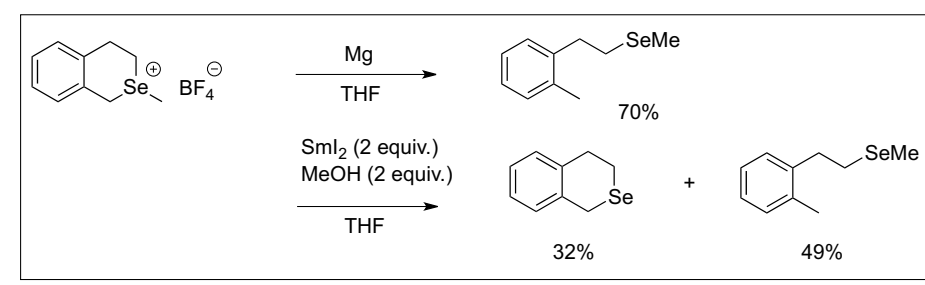

Scheme 12

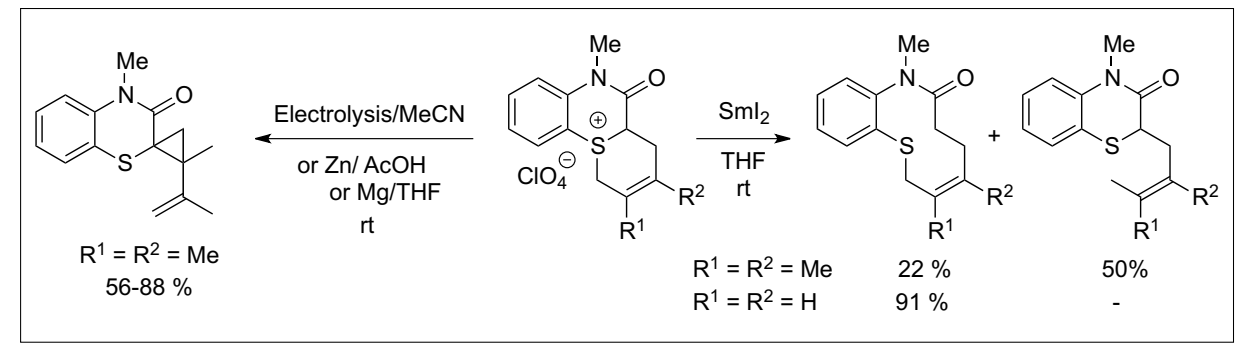

Scheme 13.

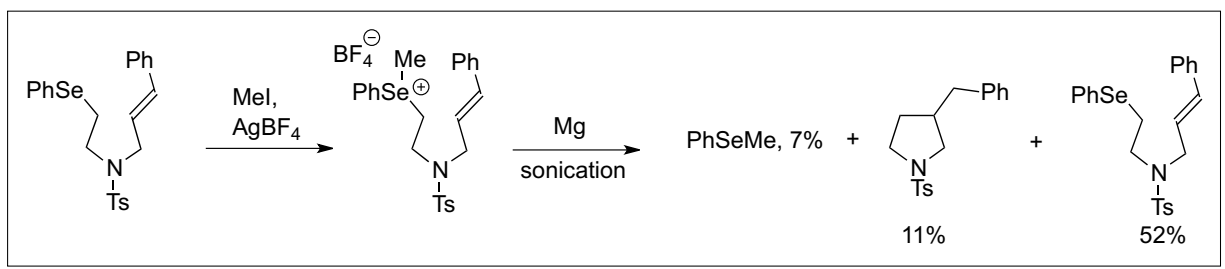

Scheme 14.

\begin{tabular}{|c|c|c|c|c|c|}
\hline $\begin{array}{r}\stackrel{\oplus}{\ominus} \mathrm{Tol}_{3} \mathrm{~S} \mathrm{PF}_{6}\end{array}$ & + & $\mathrm{PPh}_{3}$ & $\begin{array}{l}\underset{200 \mathrm{~W}}{\mathrm{hv}} \\
\text { flood lamp }\end{array}$ & $\mathrm{Tol}_{2} \mathrm{~S}$ & + TolPPh $_{3}^{\oplus} \mathrm{PF}_{6}$ \\
\hline$\dot{\mathrm{TolPPh}}_{3}$ & + & $\mathrm{Tol}_{3} \mathrm{~S}^{\oplus}$ & $\longrightarrow$ & $\stackrel{\oplus}{\text { TolPPh }_{3}}$ & $+\mathrm{Tol}_{3} \mathrm{~S}^{\cdot}$ \\
\hline & & $\mathrm{Tol}_{3} \mathrm{~S}^{\circ}$ & $\longrightarrow$ & $\mathrm{Tol}_{2} \mathrm{~S}$ & $+\mathrm{Tol}^{\circ}$ \\
\hline Tol & + & $\mathrm{PPh}_{3}$ & $\longrightarrow$ & ToIPPh $_{3}$ & \\
\hline
\end{tabular}

Scheme 15.

It relies on the use of trivalent organophosphorus compounds as mediators and corresponds to $\mathrm{S}_{\mathrm{RN}} 1$ reaction. After photoinitiation, which was accelerated by the use of the addition of phenylazoisobutyronitrile, a phosphoranyl radical is produced from the addition of an aryl radical. The latter is oxidized by the sulfonium to generate a phosphonium generating a sulfuranyl radical which falls apart. ${ }^{[59]}$

The homolytic reduction of sulfonium is a rather complex molecular process. A main issue which remains to be addressed is the control of the regioselectivity. This could be accomplished by the invention of new sets of reagents and/or reaction conditions.

\section{Homolytic Reduction of Phosphoniums}

Reductive cleavage of the carbonphosphorus bond of phosphonium salts in carbon-centered radicals has received only scant attention. Most of studies were published during the sixties followed by other sporadic reports until 1993. They showed that quaternary phosphonium cations can be reduced under electrochemical or photochemical conditions as well as by pulse radiolysis. But applications to synthesis and material science are still almost nonexistent.

A pioneer work of Colichman examined the polarographic reduction of 
phenyltrimethylphosphonium and tetra- $o$ tolylphosphonium iodides. Experiments indicated that the first one proceeds in two successive reversible one-electron steps while the second one occurs by a single two-electron reversible process. ${ }^{[60]}$ Later, Horner and his co-workers investigated the electrolysis of a series of substituted phosphonium in protic media. The carbonphosphorus bond cleavage ability and selectivity have been evaluated and appeared to depend on the nature of carbon-leaving group attached to the phosphorus atom, relating to the $\mathrm{C}-\mathrm{P}$ bonding strength, and the electrode material using either a mercury or a lead cathode. For instance, reduction of methyltriphenylphosphonium bromide at the mercury cathode gave rise to $83 \%$ of methyldiphenylphosphine (and 5.5\% of triphenylphosphine) whereas a 53:40 mixture of triphenylphosphine/methyldiphenylphosphine was obtained at the lead cathode. Experimental studies at the mercury cathode determined that the scale of ease of carbon-phosphorus bond cleavage follows the trend: benzyl, $\mathrm{HOCH}_{2} \mathrm{CH}_{2}$, tert-butyl $>i$-propyl $>n$-butyl $>$ ethyl $>$ phenyl $>$ methyl, that matches well with the stability of the corresponding radi$\mathrm{cal}^{[61]}$ and investigations on structure/reactivity relationships for unsymmetrical tetraarylphosphonium have also been performed. ${ }^{[62]}$ As an application, optically active quaternary phosphonium salts were reduced to the corresponding phosphines with retention of the configuration at the phosphorus center.[63] Then, Horner estimated the half-wave reduction potentials of differently substituted phosphoniums by polarography, which led to a similar classification. ${ }^{[64]}$ Results of Grimshaw and Ramsey showed a linear relationship between the half-wave reduction potentials and the Hammett substituent values of a wide range of benzyltriphenylphosphonium chlorides substituted on the benzyl group. ${ }^{[65]}$ In a plausible mechanism, formation of a transient phosphoranyl radical obtained by an initial monoelectronic reduction of the amalgamated phosphonium is proposed at the first step. Then, a second electron transfer followed by a protonation should generate a tetrasubstituted hydrophosphoran intermediate which may decompose by hydrogenolysis of the $\mathrm{C}-\mathrm{P}$ bond. Alternatively, the phosphoranyl radical intermediate may evolve through homolytic cleavage of a $\sigma$-carbon-phosphorus bond ( $\alpha$-elimination) releasing a new carbon-centered radical which can be reduced a second time to a carbanion and then protonate (Scheme 16). ${ }^{[64]}$

Evidence for phosphoranyl radical formation and its decomposition to free or amalgamated alkyl radicals were demonstrated by Wagenknecht and Baizer during the electroreduction of $\omega$-cyano

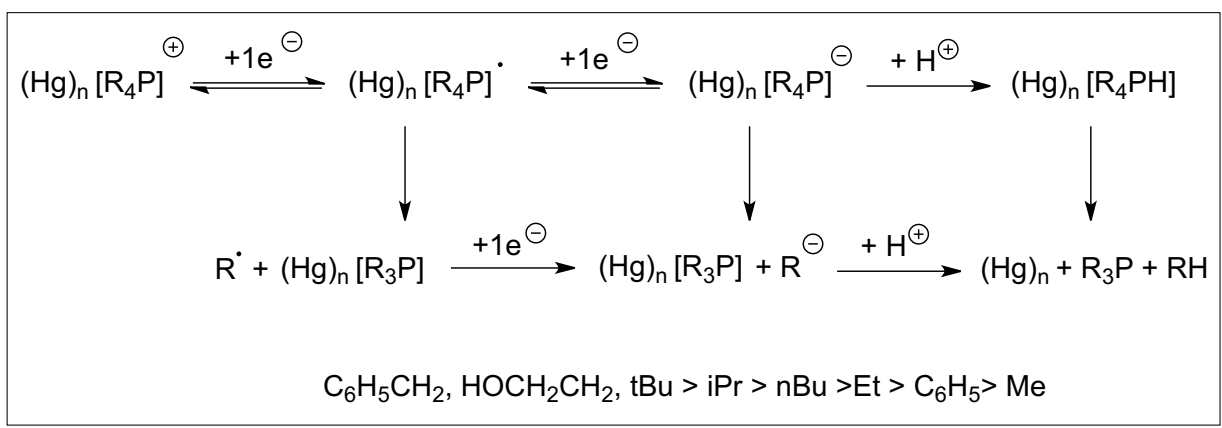

Scheme 16.

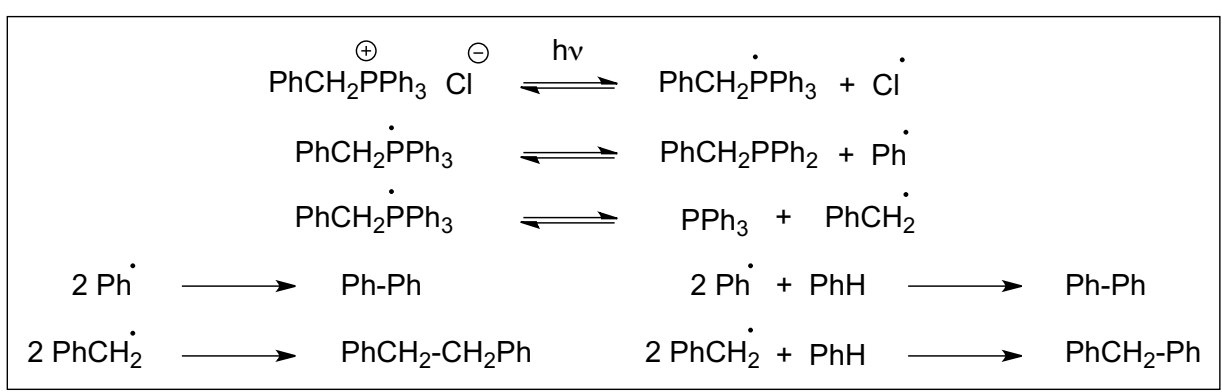

Scheme 17.

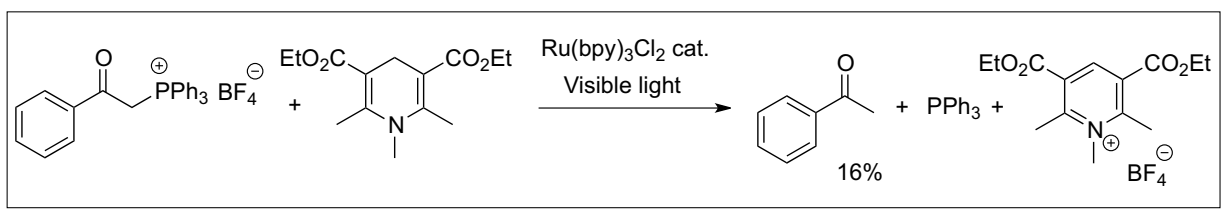

Scheme 18.

alkyltriphenylphosphonium salts at the mercury cathode in non protic media such as DMSO or DMF in the presence of styrene. Particularly, electrolysis at the platinum cathode provides free radical polymerization of styrene in excess. ${ }^{[66]}$ Finally, Savéant and Binh extended this study to the reduction of several alkyltriphenylphosphonium halides in non protic media. Various products have been identified as resulting from two different types of electron transfer: a one-electron pathway leading to dimerization and/or disproportionation of the released radical or a twoelectron pathway providing the formation of ylides, phosphine oxides or Hofmann degradation products. ${ }^{[67]}$ Interestingly, Kampmeier and Nalli reported that phosphoranyl radical intermediates generated by arylation of triphenylphosphine can act as reducing agents with iodonium and sulfonium salts. ${ }^{59]}$

Along with these electrochemical studies, photolysis of phosphonium salts was investigated by Griffin and Kaufman (Scheme 17). UV Irradiation of benzyltriphenylphosphonium chloride in benzeneethanol led to a mixture of diphenyl, diphenylmethane, dibenzyl, diphenylphosphine, triphenylphosphine and oxidation products. A mechanism involving a photoinduced electron transfer between the ben- zyltriphenylphosphonium and the chloride is assumed. After $\alpha$-scission of the phosphoranyl intermediate, both expelled benzyl and aryl radicals can dimerize and/or react with benzene as solvent. ${ }^{68]}$

In 1978, Kellog reported the reduction of phenacyl triphenylphosphonium tetrafluoroborate with Hantzsch ester under visible irradiation (Scheme 18). The brief report shows that the reaction requires the presence of $\mathrm{Ru}$ (bipy) ${ }_{3} \mathrm{Cl}_{2}$ as a photocatalyst to obtain acetophenone in $16 \%$ yield. [54] No reduction was observed under simple thermal conditions.

Applications of pulse radiolysis to the reduction of benzyltriphenylphosphonium and variously substituted phenylmethylphosphoniums in aqueous solution were proposed by Horii et al. and supported the formation of phosphoranyl and benzyl radicals among others. ${ }^{[69]}$

\section{Homolytic Reduction of Iodoniums}

The past few years have witnessed a growth in the use of iodonium salts in organic synthesis and particularly as a tool for polymerization initiation and materials surface functionalization in exploiting their ability to generate radicals. ${ }^{[70]}$ Homolytic 
reduction of iodoniums salts was in fact examined by using either electrochemical, photochemical methods or SET reagents.

Since the initial studies of Zappi and Mastropaolo, ${ }^{[71]}$ the electroreduction of symmetrically substituted iodoniums salts was investigated by Colichman and Maffei, ${ }^{[72]}$ then soon joined by the groups of Beringer ${ }^{[73]}$ and Grimshaw. ${ }^{[74]}$ No less than five different mechanisms were proposed for the reduction at a mercury cathode of diphenyliodonium cations which supported the generation of a supposed diaryliodine radical through a one-electron transfer reaction, its absorption to the electrode, and then the formation of mercurycontaining species such as the phenyl mercury radical and the diphenyl mercury, the disproportionation adduct. An alternative decomposition of the transient diaryliodine radical to iodobenzene and the phenyl free radical cannot be ruled out. For instance, in the reduction of the dibenziodolium ion, the corresponding diarylmercury and a small amount of iodo biphenyl resulting from the direct reduction of the corresponding free radical were detected. ${ }^{\text {[75] }}$ Electrolysis of unsymmetrically substituted diphenyliodonium salts was also taken into consideration by Peters. Several factors on the $\mathrm{C}-\mathrm{I}$ bond cleavage have been studied including product distribution, steric and electronic effects. Discussions on mechanistic aspects came to approximately similar conclusions. ${ }^{[76]}$

Chemical methods involving metallic reagents were investigated for the reduction of iodonium organic compounds. Metal salts such as copper(I), ${ }^{[77]}$ titanium(III) and chromium(II) chlorides were reported to trigger the decomposition of diphenyliodonium cations and liberate benzene, chlorobenzene and biphenyl as products in variable ratios, depending on the nature of the metal and the solvent used (water or methanol). While a concerted mechanism is suggested for the selective formation of chlorobenzene with $\mathrm{CuCl}$, a stepwise mechanism consistent with the experimental data is operating with $\mathrm{TiCl}_{3}$ and $\mathrm{CrCl}_{2}$. The latter may involve an initial SET process generating of free phenyl radicals by decomposition of the transient diphenyliodine radical followed by competitive $\mathrm{H}$-abstraction, ligand-radical transfer with metal salts and dimerization. ${ }^{[78]}$ Thus, the reactivity of iodonium salts toward chromium dichloride was applied by Ochiai to reactions of diaryl-, alkenyl(aryl)- and alkynyl(aryl)iodonium tetrafluoroborates with aldehydes. For instance, treatment of (o-allyloxyphenyl)mesityliodonium salt with three equivalents of $\mathrm{CrCl}_{2}$ in the presence of five equivalents of benzaldehyde afforded the substituted dihydrobenzofuran in $47 \%$ yield (Scheme 19). Presumably, the aryl radical generated through homo-

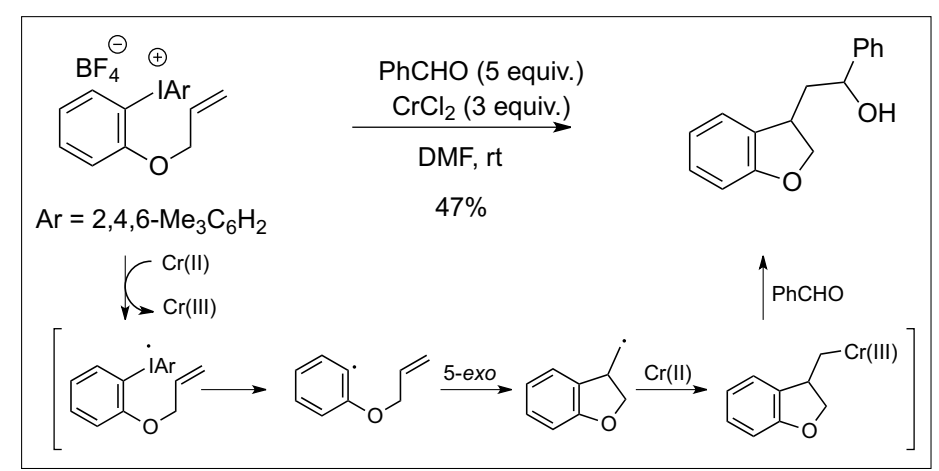

Scheme 19.

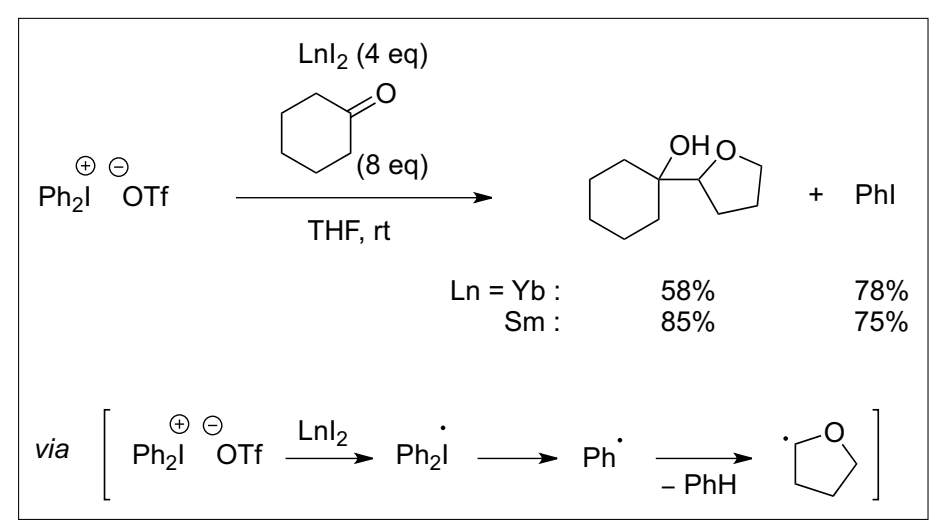

Scheme 20

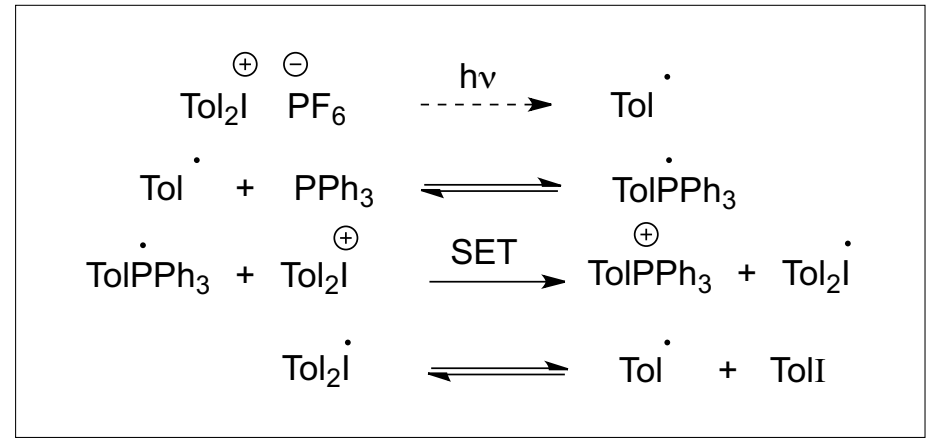

Scheme 21

lytic reduction of the iodonium undergoes a 5-exo cyclization. The resulting primary radical reacts with a second chromium(II) ion providing an alkylchromium species which adds to benzaldehyde. ${ }^{779]}$

Low-valent lanthanide reagents such as ytterbium and samarium diiodide were shown to be efficient catalysts for the reduction of diphenyliodoniums to phenyl free radicals and iodobenzene. When the reaction is conducted in THF, the phenyl radical abstracts a hydrogen atom from THF. Then, the tetrahydrofuranyl radical is captured by divalent $\mathrm{LnI}_{2}(\mathrm{Ln}=\mathrm{Yb}$ or $\mathrm{Sm}$ ) and the resulting organometallic compound adds to the ketone (Scheme 20). ${ }^{[80]}$

Photo-excited electron transfer processes have also been considered to promote the reduction of diphenyliodonium ions. In 1993, Kampmeier and Nalli reported the photodecomposition of diaryliodoniums with triphenylphosphine involving a radical chain mechanism (Scheme 21). Photoirradiation of a diaryliodoiodonium salt liberates an aryl radical which is trapped by triphenylphosphine and generates a phosphoranyl radical intermediate. A single-electron transfer between the iodonium salt and the phosphoranyl radical occurs and gives rise to an arylphosphonium and a diaryliodine radical. The latter collapses to an iodoaryl and an aryl radical which can propagate the chain reaction. ${ }^{[59]}$ The same reaction performed in THF in the absence of triphenylphosphine liberates arenes and iodoarenes. In this case, the role of the radical mediator is ensured by the tetrahydrofuranyl radical generated from aryl radical $\mathrm{H}$-abstraction at THF. [81]

Electron transfer photosensitization of diaryliodonium salts by either organic dyes or photocatalysts such as polypyridine ruthenium or iridiumcomplexes have been mostly used for initiation of free radical polymerization and cationic polymerization. ${ }^{[82]}$ For instance, Lalevée recently published a new photocatalytic system composed by the tris(2,2'-bipyridine)ruthenium(II) complex (or an iridium (III) complex), a 


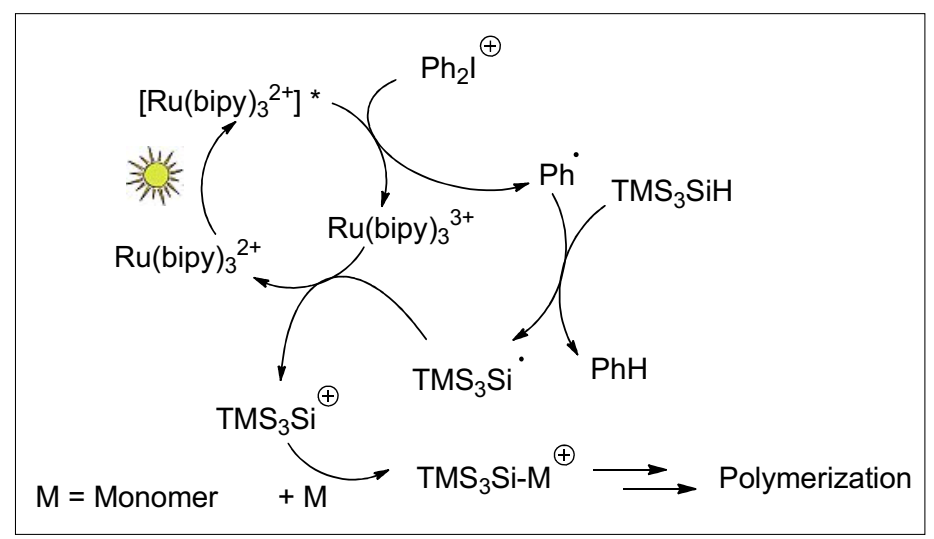

Scheme 22

diphenyliodonium salt and a silane for the photoinitiation of cationic polymerization of epoxydes (Scheme 22). Reduction of diphenyliodonium by the photoactivated $\left[\mathrm{Ru} \text { (bipy) }{ }_{3}{ }^{2+}\right]^{*}$ releases a phenyl radical that can abstract a hydrogen atom from the silane. Electron transfer reaction between the formed silyl radical and another molecule of diphenyliodonium generates a silylium which can promote the cationic polymerization of epoxydes. ${ }^{[83]}$

\section{Conclusion and Perspectives}

This overview covers the homolytic reduction of diazonium, sulfonium, selenonium, telluronium, phosphonium and iodonium salts to generate $\mathrm{C}$-centered radicals (alkyl or aryl). The latter can be conveniently engaged to participate in subsequent synthetic transformations, generally inter- or intramolecular additions. Several versatile devices are now at hand to accomplish this task, relying mainly on electrochemical and photochemical methods or single-electron transfer (SET) reagents. An interesting trend in the chemical reduction of onium salts is the possibility to operate catalytic processes involving visible light activation. Our laboratory is active in this field and our results will be reported in due course. ${ }^{[84]}$

Received: May 8, 2012

[1] a) 'The Chemistry of the Sufonium Group', Ed. C. M. Stirling, Wiley, New York, 1981; b) 'The Chemistry of Organophosphorus Compounds, vol. 3: Phosphonium Salts, Ylides and Phosphoranes', Ed. R. Hartley, John Wiley \& Sons, Ltd. New York, 1994; c) 'Onium Ions', Eds. G. A. Olah, K. K. Laali, G. K. Wang, Q Parkash Surya, John Wiley \& Sons Ltd, US, 1998.

[2] a) T. Sandmeyer, Ber. Dtsch. Chem. Ges. $\mathbf{1 8 8 4}$ 17, 1633; b) T. Sandmeyer, Ber. Dtsch. Chem. Ges. 1884, 17, 2650 .

[3] H. H. Hodgson, Chem. Rev. 1947, 40, 251.

[4] R. Pschorr, Chem. Ber. 1896, 29, 496.

[5] M. Gomberg, W. E. Bachmann, J. Am. Chem. Soc. 1924, 42, 2339.

[6] H. Meerwein, E. Buchner, K. van Emsterk, $J$ Prakt. Chem. 1939, 152, 237.
[7] M. R. Heinrich, Chem. Eur. J. 2009, 15, 820

[8] For the Meerwein reaction: J. K. Kochi, J. Am. Chem. Soc. 1956, 78, 1228.

[9] For the Sandmeyer reaction: J. K. Kochi, J. Am. Chem. Soc. 1957, 79, 2942

[10] H. Zollinger, Acc. Chem. Res. 1973, 6, 335.

[11] M. P. Doyle, B. Siegfried, R. C. Elliott, J. F. Dellaria, Jr., J. Am. Chem. Soc. 1977, 42, 2431.

[12] A. Citterio, F. Minisci, E. Vismara, J. Org. Chem. 1982, 47, 81

[13] A. L. J. Beckwith, R. O. C. Norman, J. Chem. Soc. B. 1969, 403 .

[14] N. A. Porter, G. R. Dubay, J. G. Green, J. Am. Chem. Soc. 1978, 100, 920.

[15] E. M. Kosower, Acc. Chem. Res. 1971, 4, 193.

[16] E. Bonfand, L. Forslund, W. B. Motherwell, S. Vazquez, Synlett 2000, 475

[17] M. R. Heinrich, O. Blank, S. Wölfel, Org. Lett. 2006, $8,3323$.

[18] M. R. Heinrich, A. Wetzel, M. Kirschstein, Org. Lett. 2007, 9, 3833.

[19] C. de Salas, O. Blank, M. R. Heinrich, Chem. Eur. J. 2011, 17, 9306.

[20] M. R. Heinrich, O. Blank, D. Ullrich, M. Kirschstein, J. Org. Chem. 2007, 72, 9609.

[21] A. Wetzel, V. Ehrhardt, M. R. Heinrich, Angew. Chem. Int. Ed. 2008, 47, 9130.

[22] C. Graebe, F. Ullman, Chem. Ber. 1894, 27, 3483.

[23] A. H. Lewin, T. Cohen, J. Org. Chem. 1967, 32, 3844

[24] A. H. Lewin, R. J. Michl, J. Org. Chem. 1973, $38,1126$.

[25] A. H. Lewin, N. C. Peterson, R. J. Michl, J. Org. Chem. 1974, 39, 2747.

[26] H. Cano-Yelo, A. Deronzier, J. Chem. Soc., Perkin Trans. 2 1984, 1093

[27] H. Cano-Yelo, A. Deronzier, Tetrahedron Lett. 1984, 25, 5517.

[28] H. Cano-Yelo, A. Deronzier, New J. Chem. 1987, 11, 479.

[29] D. Kalyani, K. B. McMurtrey, S. R. Neufeldt, M. S. Sanford, J. Am. Chem. Soc. 2011, 133, 18566.

[30] D. P. Hari, P. Schroll, B. König, J. Am. Chem. Soc. 2012, 134, 2958.

[31] J. A. Murphy, C. Lampard and N. Lewis, J. Chem. Soc., Chem. Commun., 1993, 295.

[32] J. A. Murphy, in 'Radical in Organic Synthesis', Eds. P. Renaud, M. P. Sibi, Wiley-VCH, Weinheim, Germany, 2001, Vol. 1, 298.

[33] T. Koizumi, N. Bashir, J. A. Murphy, Tetrahedron Lett. 1997, 38, 7635.

[34] T. Koizumi, N. Bashir, A. R. Kennedy, J. A Murphy, J. Chem. Soc., Perkin Trans. 1, 1999, 3637.

[35] O. Callaghan, C. Lampard, A. R. Kennedy, J. A. Murphy, J. Chem. Soc., Perkin Trans. 1 1999, 995.

[36] M. Mahesh, J. A. Murphy, F. LeStrat, H. P. Wessel, Beilstein J. Org. Chem. 2009, 5, 1 .

[37] N. Kornblum, G. D. Cooper, J. E. Taylor, J. Am. Chem. Soc. 1950, 72, 3013.
[38] W. T. Evanochko, P. B. Shevlin, J. Am. Chem. Soc. 1979, 101, 4668.

[39] A. N. Abeywickrema, A. L. J. Beckwith, J. Org. Chem. 1987, 52, 2568 and references cited herein.

[40] a) C. E. Farrar, K. K. W. Siu, H. P. Lynne, J. T. Jarrett, Biochemistry 2010, 49, 9985; b) M Fontecave, J.-L. Pierre, C. R. Acad. Sci. Paris, Chimie / Chemistry 2001, 4, 531; c) S. C. Wang, P. A. Frey, Trends in Biochemical Sciences 2007, 32, 101.

[41] For a study on the regioselectivity in the homolytic cleavage of SAM, see: J. A. Kampmeier, Biochemistry 2010, 49, 10770.

[42] K. H. Vase, A. H. Holm, K. Norrman, S. U. Pedersen, K. Daasbjerg, Langmuir, 2008, 24 182.

[43] M. Bonifacic, E. Anklam, J. Chem. Soc., Perkin Trans. 2 1991, 243.

[44] P. Eriksson, L. Engman, J. Lind, G. Merényi, Eur. J. Org. Chem. 2005, 701.

[45] a) A. Ghanimi, J. Simonet, New J. Chem. 1997, 21, 257; b) T. Kjaersbo, K. Daasbjerg, S. U. Pedersen, Electrochemica Acta 2003, 48, 1807.

[46] a) P. Martigny, J. Simonet, G. Mousset, J. Vigneron, Nouv. J. Chimie 1983, 7, 299; b) F. D. Saeva; B. P. Bradley, J. Am. Chem. Soc. 1984, 106, 4121; c) P. Martigny, J. Simonet, J. Electroanal. Chem. 1979, 101, 275; d) A. Stasko, P. Rapta, V. Brezova, O. Nuyken, R. Vogel, Tetrahedron 1993, 49, 10917.

[47] a) L. Pause, M. Robert, J.-M. Savéant, J. Am. Chem. Soc. 2001, 123, 4886; b) C. P. Andrieux, M. Robert, F. D. Saeva, J.-M. Savéant, J. Am. Chem. Soc. 1994, 116, 7864.

[48] J. A. Kampmeier, AKM M. Hoque, F. D. Saeva, D. K. Wedegaertner, P. Thomsen, S. Ullah, J. Krake, T. Lund, J. Am. Chem. Soc. 2009, 131, 10015.

[49] a) F. D. Saeva, Tetrahedron 1986, 42, 6123; b) R. Schwalm, R. Bug, G.-S. Dai, P. M. Fritz, M. Reinhardt, S. Schneider, W. Schnabel, J. Chem. Soc., Perkin Trans 2 1991, 1803.

[50] L. Dektar, N. P. Hacker, J. Am. Chem. Soc. 1990, 112, 6004 .

[51] S. L. Nickol, J. A. Kampmeier, J. Am. Chem. Soc. 1973, 95, 1908.

[52] X. Wang, F. D. Saeva, J. A. Kampmeier, J. Am. Chem. Soc. 1999, 121, 4364.

[53] a) F. D. Saeva, D. T. Breslin, H. R. Luss, J. Am. Chem. Soc. 1991, 113, 5333; b) F. D. Saeva, P. A. Martic, E. Garcia, J. Phys. Org. Chem. 1993, $6,333$.

[54] a) D. M. Hedstrand, W. H. Kruizinga, R. M Kellogg, Tetrahedron Lett. 1978, 1255; b) T. J. van Bergen, W. H. Kruizinga, R. M. Kellogg, $J$. Org. Chem. 1979, 44, 4953.

[55] S.-K. Chung, K. Sasamoto, Chem. Commun. 1981, 346.

[56] P. Beak, T. A. Sullivan, J. Am. Chem. Soc. 1982 104, 4450 .

[57] a) M. Hori, T. Kataoka, H. Shimizu, K Tsutsumi, Tetrahedron Lett. 1989, 30, 981; b) T. Kataoka, K. Tsutsumi, T. Iwama, H. Shimizu, M. Hori, Tetrahedron Lett. 1990, 31, 3027; c) T. Kataoka, T. Iwama, H. Shimizu, M. Hori, Phosphorus, Sulfur and Silicon 1992, 67, 169; d) T. Kataoka, K. Tsutsumi, K. Kano, K. Mori, M. Miyake, M. Yokota, H. Shimizu, M. Hori, J. Chem. Soc., Perkin Trans. 1990, 3017.

[58] T. Kataoka, Y. Nakamura, H. Matsumoto, T. Iwama, H. Kondo, H. Shimizu, O. Muraoka, G. Tanabe, J. Chem. Soc., Perkin Trans. 11997 309.

[59] J. Kampmeier, T. W. Nalli, J. Org. Chem. 1993 $58,943$.

[60] E. L. Colichman, Anal. Chem. 1954, 26, 1204.

[61] L. Horner, A. Mentrup, Liebigs Ann. Chem. 1961, 646, 65.

[62] L. Horner, J Haufe, Chem. Ber. 1968, 101, 2903.

[63] L. Horner, H. Fuchs, H. Winkler, A. Rapp, Tetrahedron Lett. 1963, 4, 965. 
[64] L. Horner, F. Röttger, H. Fuchs, Chem. Ber. 1963, 96, 3141.

[65] J. Grimshaw, J. S. Ramsey, J. Chem. Soc. B 1968, 63.

[66] J. H. Wagenknecht, M. M. Baizer, J. Org. Chem. 1966, 31, 3885.

[67] J. M. Savéant, S. K. Binh, J. Org. Chem. 1977, $42,1242$.

[68] C. E. Griffin, M. L. Kaufman, Tetrahedron Lett. 1965, 6, 773

[69] a) H. Horii, S. Fujita, T. Mori, S. Taniguchi, Bull. Chem. Soc. Jpn. 1979, 52, 3099; b) H. Horii, S. Fujita, T. Mori, S. Taniguchi, Radiat. Phys. Chem. 1982, 19, 231.

[70] M. S. Yusubov, A. V. Maskaev, V. V. Zhdankin, ARKIVOC 2011, 370 and references cited herein.

[71] E. V. Zappi, R. F. Mastropaolo, Anales Asoc. Quim. Arg. 1941, 29, 88.

[72] E. L. Colichman, H. P. Maffei, J. Am. Chem. Soc. 1952, 74, 2744.

[73] a) H. E. Bachofner, F. M. Beringer, L. Meites, J. Am. Chem. Soc. 1958, 80, 4269 and 4274; b) H
E. Bachofner, F. M. Beringer, L. Meites, J. Am. Chem. Soc. 1958, 80, 4274.

[74] J. A. Azoo, F. G. Coll, J. Grimshaw, J. Chem. Soc. C 1969, 2521.

[75] F. M. Beringer, S. Messing, J. Org. Chem. 1972, 37, 2484.

[76] M. S. Mubarak, D. G. Peters, J. Org. Chem. 1985, 50, 673 .

[77] F. M. Beringer, E. J. Geering, I. Kuntz, M. Mausner, J. Phys. Chem. 1956, 60, 141.

[78] F. M. Beringer, P. Bodlaender, J. Org. Chem. 1969, 34, 1981

[79] D.-W. Chen, M. Ochiai, J. Org. Chem. 1999, 64, 6804.

[80] Y. Makioka, Y. Fujiwara, T. Kitamura, J. Orgmet. Chem 2000, 611, 509.

[81] J. Kampmeier, T. W. Nalli, J. Org. Chem. 1994, 59, 1381.

[82] a) J. V. Crivello, in 'Photoinitiators for Free Radical, Cationic and Anionic Photopolymerization, 2nd ed.', Ed. G. Bradley, John Wiley and Sons, New York, 1998; b) J. P. Fouassier, 'Photoinitiation,
Photopolymerization and Photocuring: Fundamental and Applications', Hanser Publishers, New York, 1995; c) K. D. Belfield, J. V. Crivello, 'Photoinitiated polymerization', ACS Symposium Series 847, American Chemical Society, Washington, DC, 2003; d) J. P. Fouassier, J. Lalevée, 'Photoinitiators for Polymer Synthesis: Scope, Reactivity and Efficiency', Wiley-VCH, Weinheim, Germany, to appear in $\mathbf{2 0 1 2}$.

[83] a) J. Lalevée, N. Blanchard, M.-A. Tehfe, F. Morlet-Savary, J.-P. Fouassier, Macromolecules 2010, 43, 10191; b) J. Lalevée, M. Peter, F. Dumur, D. Gigmes, N. Blanchard, M.-A. Tehfe, F. Morlet-Savary, J.-P. Fouassier, Chem. Eur. J. 2011, 17, 15027 and references cited herein.

[84] a) M.-H. Larraufie, R. Pellet, L. Fensterbank, J.P. Goddard, E. Lacôte, M. Malacria, C. Ollivier, Angew. Chem. Int. Ed. 2011, 50, 4463; b) L. Fensterbank, invited lecture at the 5th Pacific Symposium on Radical Chemistry, Shirahama, Japan, 2011. 